\title{
Photoluminescence study of interband transitions in few-layer, pseudomorphic, and strain-unbalanced Ge/GeSi multiple quantum wells
}

\author{
M. Montanari, ${ }^{1,2,{ }^{*}}$ M. Virgilio, ${ }^{3}$ C. L. Manganelli, ${ }^{2}$ P. Zaumseil,${ }^{2}$ M. H. Zoellner, ${ }^{2}$ Y. Hou, ${ }^{2}$ M. A. Schubert ${ }^{2}$ \\ L. Persichetti, ${ }^{1}$ L. Di Gaspare, ${ }^{1}$ M. De Seta, ${ }^{1}$ E. Vitiello, ${ }^{4}$ E. Bonera, ${ }^{4}$ F. Pezzoli, ${ }^{4}$ and G. Capellini ${ }^{1,2}$ \\ ${ }^{1}$ Dipartimento di Scienze, Università degli Studi Roma Tre, Viale Marconi 446, I-00146 Roma, Italy \\ ${ }^{2}$ IHP - Leibniz-Institut für innovative Mikroelektronik, Im Technologiepark 25, D-15236 Frankfurt (Oder), Germany \\ ${ }^{3}$ Dipartimento di Fisica E. Fermi, Università di Pisa, L.go Pontecorvo 3, I-56127 Pisa, Italy \\ ${ }^{4}$ L-NESS, Università di Milano-Bicocca, Via R. Cozzi 55, 20125 Milano, Italy
}

(Received 11 September 2018; revised manuscript received 24 October 2018; published 19 November 2018)

\begin{abstract}
In this paper we investigate the structural and optical properties of few strain-unbalanced multiple $\mathrm{Ge} / \mathrm{GeSi}$ quantum wells pseudomorphically grown on GeSi reverse-graded substrates. The obtained high epitaxial quality demonstrates that strain symmetrization is not a mandatory requirement for few quantum-well repetitions. Photoluminescence data, supported by a thorough theoretical modeling, allow us to unambiguously disentangle the spectral features of the quantum wells from those originating in the virtual substrate and to evaluate the impact on the optical properties of key parameters, such as quantum confinement, layer compositions, excess carrier density, and lattice strain. This detailed understanding of the radiative recombination processes is of paramount importance for the development of Ge/GeSi-based optical devices.
\end{abstract}

DOI: 10.1103/PhysRevB.98.195310

\section{INTRODUCTION}

$\mathrm{Ge} / \mathrm{GeSi}$ multiple quantum wells (MQWs) have attracted great interest for Si-based photonic devices since the demonstration of the quantum confined Stark effect at room temperature (RT) [1]. Optical modulators [2-6], photodetectors [7-9], and spin-based optoelectronic concepts [10-13] have been investigated using this promising material. Furthermore, RT direct-gap emission has been observed by means of electroluminescence [14] and photoluminescence (PL) [15], indicating that $\mathrm{Ge} / \mathrm{GeSi} \mathrm{MQW}$ are potential candidates for an efficient silicon-compatible light emitter. A step further, necessary to exploit Ge/GeSi MQWs as the active medium in a laser, is to obtain positive net optical gain.

Recently, strain engineering has been proposed to achieve optical gain in Ge/GeSi MQWs [16]. Among the several methods investigated to induce external tensile strain in Ge [17-23], strategies relying on a silicon nitride external stressor [24-26] are very promising since, in principle, any arbitrary stress can be transferred. Moreover, the combination of external strain and quantum confinement results in two independent parameters to tune the emission wavelength by design, enabling the realization of light emitters of different "colors" integrated on the same chip. The drawback is that, to minimize the detrimental effect on the emission properties due to an inhomogeneous vertical strain distribution [27,28], the thickness of the active region should be limited to a few hundred nanometers. It follows that, in order to expand this strategy to MQWs systems, a limited number of QWs needs to be grown.

*Corresponding author: michele.montanari@uniroma3.it
To the best of our knowledge, PL of a few periods of Ge-rich Ge/GeSi MQWs has been demonstrated only on samples grown directly on Ge substrates [29,30]. Unfortunately, heteroepitaxial strain, arising from the $4.2 \%$ lattice mismatch between $\mathrm{Ge}$ and $\mathrm{Si}$, results in crystal defects, such as threading dislocations, that behave as nonradiative recombination centers and their presence is then detrimental for the efficiency of optoelectronic devices [31,32]. To achieve high- $x \mathrm{Ge}_{x} \mathrm{Si}_{1-x}$ layers with low threading dislocations density, relatively thick GeSi reverse-graded virtual substrates (RG-VSs) where the lattice mismatch is gradually distributed among several layers are commonly used [33-36]. Employing so many substrate layers with different concentrations results in complicated PL spectra. This is true, in particular, if a long-wave pump is used for homogeneous excitation and the number of QWs is limited to few repetitions since both the MQW region and the underlying layers are simultaneously excited. Indeed, in this case a one-to-one identification of all the individual spectral components is not trivial, especially if complementary techniques, such as optical absorption, are not employed. Thus, for the application of external stressors on a few QWs grown on $\mathrm{GeSi}$ RG-VSs, it is necessary to first systematically characterize the unstressed structures and develop a model to unambiguously assess the optical properties of the Ge MQWs, isolating the emission features due to the excited region of the substrate.

Here, we present a paper on the optical properties of undoped unstressed Ge MQWs surrounded by Ge-rich GeSi barriers, grown on relatively thin $(\lesssim 2.5-\mu \mathrm{m})$ RG-VSs. The high quality of the samples as probed by high-resolution transmission electron microscopy, x-ray diffraction (XRD), and atomic force microscopy (AFM) and the good agreement between the observed and simulated data allowed us to unambiguously interpret the emission spectra. 
TABLE I. Material parameters of the investigated samples as determined by TEM and XRD. Samples are labeled as $\mathrm{S}$ thickness of the $Q W$ number of periods.

\begin{tabular}{|c|c|c|c|c|c|c|}
\hline $\mathrm{Ge}$ & 700 & 0 & 0 & & & \\
\hline VS & 700 & $1.8 \mu \mathrm{m}$ & 0 & & & \\
\hline S10-10 & 700 & $1.8 \mu \mathrm{m}$ & 10 & $10.5+17.2=27.7(27.5)$ & -0.6 & 0.1 \\
\hline S10-5 & 700 & $1.8 \mu \mathrm{m}$ & 5 & $10.9+17.1=28.0(28.1)$ & -0.6 & 0.1 \\
\hline S17-5 & 700 & $1.8 \mu \mathrm{m}$ & 5 & $17.1+14.7=31.8(31.5)$ & -0.5 & 0.1 \\
\hline
\end{tabular}

\section{SAMPLE PREPARATION, EXPERIMENTAL AND THEORETICAL METHODS}

The Ge MQWs samples listed in Table I have been grown by cold-wall ultrahigh-vacuum chemical vapor deposition on $n$-Si (001) substrates from ultrapure silane $\left(\mathrm{SiH}_{4}\right)$ and germane $\left(\mathrm{GeH}_{4}\right)$ without carrier gas. The pressure during the growth was in the millitorr range, whereas the system base pressure is in the low $10^{-10}$ Torr range. After an ex situ wet-chemical cleaning, the $\mathrm{Si}$ substrates were annealed for $10 \mathrm{~min}$ in $\mathrm{H}_{2}$ environment at $1150{ }^{\circ} \mathrm{C}$ to remove the native oxide. To restore a good surface quality, a Si buffer layer has been grown at $850^{\circ} \mathrm{C}$ and $p=0.80$ mTorr. Subsequently, we have deposited a 700-nm-thick plastically relaxed Ge layer by means of a multitemperature technique [35]. The rest of the VS has been deposited at $500{ }^{\circ} \mathrm{C}$ and consists of four $\mathrm{Ge}_{x} \mathrm{Si}_{1-x}$ layers of 150-nm each, followed by a constant composition buffer layer 1.2- $\mu \mathrm{m}$ thick. The composition spanned from pure $\mathrm{Ge}(x=1)$ to the thick $\mathrm{Ge}_{0.81} \mathrm{Si}_{0.19}$ layer in $\sim 0.05 \mathrm{Ge}$ composition steps, resulting in a average Ge grading rate of $0.42 \mu \mathrm{m}^{-1}$. On top of this VS, we have deposited different Ge wells confined between nominally $\mathrm{Ge}_{0.85} \mathrm{Si}_{0.15}$ barriers with different thicknesses and/or numbers of periods. Finally, on top of the MQWs, a 30-nm-thick $\mathrm{Ge}_{0.85} \mathrm{Si}_{0.15}$ cap layer has been deposited. The growth rate of the multiquantum well region was $\sim 0.1 \mathrm{~nm} \mathrm{~s}^{-1}$.

The surface morphology of the samples was analyzed by AFM in tapping mode, whereas TEM was used to study the MQWs' structure and the VSs.

XRD measurements were carried out with a SmartLab diffractometer from Rigaku equipped with a 9-kW rotating anode $\mathrm{Cu}$ source $(\lambda=0.15406 \mathrm{~nm})$, a Ge $(400) \times 2$ crystal collimator, and a Ge $(220) \times 2$ crystal analyzer.

Microphotoluminescence ( $\mu$-PL) measurements were carried out using a custom-designed Horiba setup featuring a $50 \times$ optical microscope (numerical aperture of $A=0.65$ ), a high-resolution (HR) spectrometer optimized for IR measurements (Horiba iHR320), an extended-InGaAs detector (0.6-1.1-eV detection range), and a liquid-nitrogen Linkam cryostat allowing for varying the sample temperature from 80 up to $350 \mathrm{~K}$ with a $\pm 2-\mathrm{K}$ accuracy. A 1064-nm laser was focused on the sample surface with a spot size of about $1.7 \mu \mathrm{m}$ and an excitation power density ranging between $5.6 \times 10^{4}$ and $5.6 \times 10^{5} \mathrm{~W} \mathrm{~cm}^{-2}$. All the spectra were collected in backscattering geometry, and a white-body lamp was used to determine the optical response of the setup used for the spectra calibration.
The electronic band structure and transition energies of the Ge MQWs samples and of the different buffer layers have been calculated relying on two different theoretical frameworks: a first-neighbor tight-binding Hamiltonian model $[37,38]$ and a multivalley effective-mass description $[39,40]$. The predictivity of these two models for the evaluation of electronic spectra in GeSi multilayer heterostructures is well established [41-43], and indeed, compatible numerical results have been obtained when calculating the numerical data discussed in this paper.

\section{RESULTS AND DISCUSSION}

\section{A. Structural analysis}

Figure 1(a) shows the EDX composition profile superimposed on the corresponding TEM image for sample S17-5, ranging from the interface between the MQWs region and the $\mathrm{Ge}_{0.81} \mathrm{Si}_{0.19}$ buffer down to the Si substrate. No interdiffusion or segregation is observed within the sensitivity of the technique.

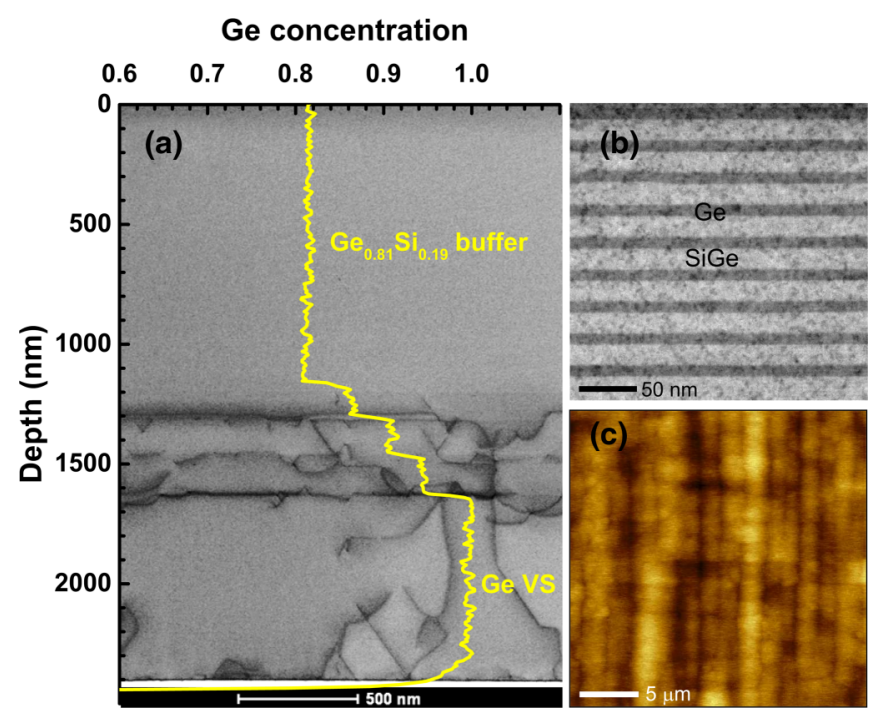

FIG. 1. (a) Energy-dispersive x-ray spectroscopy (EDX) composition profile superimposed on the TEM image for the RG-VS of sample S17-5. (b) TEM image of the QW's region of sample S10-10. (c) The $25 \times 25-\mu \mathrm{m}^{2}$ surface morphology of sample S10-10 measured with AFM. The height range is $21 \mathrm{~nm}$. The image sides are aligned along the $\langle 011\rangle$ directions. 

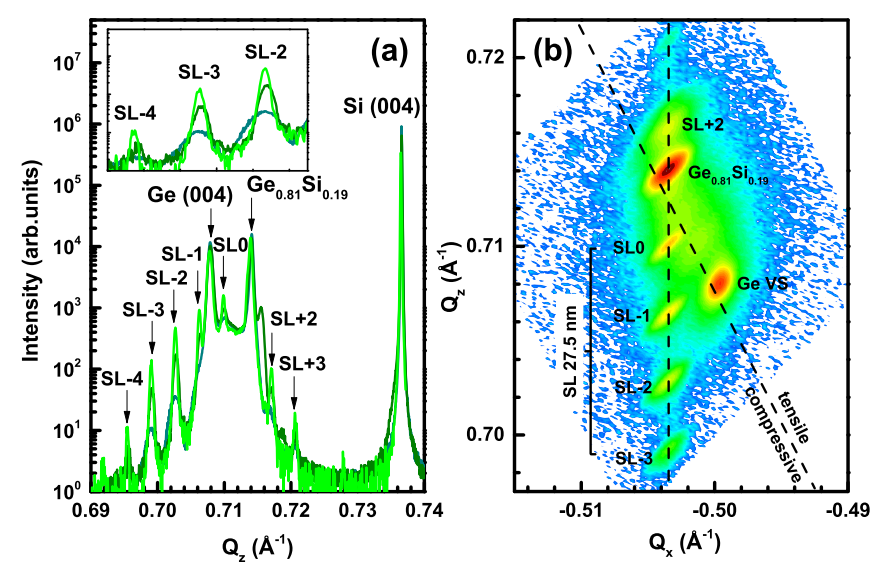

FIG. 2. (a) XRD rocking curve of samples S10-2 (dark green), S10-5 (green), and S10-10 (light green). The nominal thickness of the QWs is the same. In the inset a detail of superlattice (SL) peaks is reported. (b) Reciprocal space maps of asymmetric $(4 \overline{2} \overline{2})$ reflections of sample S10-10.

In the bottom part of the TEM image, we note the presence of extended defects, such as threading and misfit dislocations, due to the plastic relaxation of the heteroepitaxial strain $[35,44]$. The threading dislocation density of sample S10-10, obtained by etch-pit counting (not shown here), is about $1 \times 10^{7} \mathrm{~cm}^{-2}$ on the surface.

Homogeneous periodicity and abrupt barrier-well interfaces are observed in the Ge/GeSi QWs stack [Fig. 1(b)]. The thickness of the QWs and barriers, evaluated by the analysis of TEM images, is reported in Table I as $t_{w}$ and $t_{b}$, respectively. A good repeatability of the deposition process is observed, being $t_{w}$ and $t_{b}$ nominally the same for samples S10-5 and S10-10.

The lattice tilt, arising from the network of dislocations, leads to a formation of a cross-hatch pattern at the surface $[45,46]$ as shown in the AFM image reported in Fig. 1(c). The root-mean-square surface roughness is about $2.5 \mathrm{~nm}$ within a $25 \times 25-\mu \mathrm{m}^{2}$ image.

In order to determine the strain status and the actual composition of the GeSi layers, the samples were characterized by XRD rocking curves and XRD reciprocal space maps. In Fig. 2(a) we report a (004) rocking curve of samples S10-2, S10-5, and S10-10 around the (004) Ge and (004) Si Bragg peaks. The only differences among the samples is the number of QWs, the thickness of wells $\left(t_{w}\right)$, and barriers $\left(t_{b}\right)$ being nominally the same. The curve is plotted as a function of the out-of-plane scattering vector $Q_{z}=$ $4 \pi \sin (2 \Theta / 2) / \lambda$. Three main peaks are observed at scattering vectors $Q_{z} \sim 0.708, \sim 0.714$, and $\sim 0.736$, which are related to diffraction peaks from the $\mathrm{Ge}, \mathrm{GeSi}$, and $\mathrm{Si}$ layers, respectively. Multiple orders of SL satellites are observed for all the samples, indicating high crystal quality and sharp interfaces between Ge wells and GeSi barriers as also demonstrated by TEM images. The spacing between the superlattice fringes (Kiessig fringes) is inversely proportional to the periodicity of the Ge wells [46], and the spatial periodicity of the grown heterostructures obtained (27.5 nm for sample S10-10) is in good agreement with the analysis of TEM images $(27.7 \mathrm{~nm}$ for sample S10-10). For all the samples the peak positions are the same, indicating a good repeatability in the thickness of the QWs. As expected, increasing the number of periods increases the intensity of the SL peaks. XRD rocking curve measurements have been carried out on all the samples, and the spatial periodicity obtained is reported in Table I.

HR-XRD reciprocal space maps around asymmetric $(4 \overline{2} \overline{2})$ reflections are shown in Fig. 2(b). The spot corresponding to the $\mathrm{Ge}_{0.81} \mathrm{Si}_{0.19}$ buffer layer is slightly shifted from the relaxation line (i.e., the line of fully relaxed $\mathrm{GeSi}$ growth, going from $\mathrm{Si}$ to $\mathrm{Ge}$, represented by the dashed diagonal line), indicating that the layer is over-relaxed due to the difference between the coefficients of thermal expansion in $\mathrm{Ge}$ and $\mathrm{Si}$ [47]. Since the $\mathrm{Ge}_{0.81} \mathrm{Si}_{0.19}$ layer is tensile strained, its in-plane lattice parameter is equivalent to the lattice parameter of a $\mathrm{Ge}_{0.86} \mathrm{Si}_{0.14}$ relaxed bulk alloy. As a consequence, the MQWs are not strain compensated. Nevertheless, Fig. 2(b) indicates that the peaks related to the MQWs are vertically aligned to the peak of the $\mathrm{Ge}_{0.81} \mathrm{Si}_{0.19}$ buffer layer (dashed vertical line). It follows that, although the strain is not symmetrized, the entire MQW stack is coherent with the in-plane lattice parameter of the underlying VS, thanks to the small number of periods. Owing to the coherent growth, the $\mathrm{Ge}$ wells are tetragonally distorted with an in-plane lattice strain $\varepsilon_{\|}=-0.6 \pm 0.1 \%$, whereas the $\mathrm{Ge}_{0.85} \mathrm{Si}_{0.15}$ barrier lattice is slightly tensile strained, being $\varepsilon_{\|}=0.1 \pm 0.1 \%$ (see Table I).

\section{B. Optical properties}

We now discuss the $\mu$-PL properties of the investigated samples. Aiming at an unequivocal identification of the origin of the peaks in the PL spectra, we have studied the optical properties of two benchmark samples. The first is the $\mathrm{Ge} / \mathrm{Si}$ layer, and the second is the $\mathrm{Ge}_{0.81} \mathrm{Si}_{0.19}$ VS grown on top of $\mathrm{Ge} / \mathrm{Si}$ (top two lines in Table I), labeled in the following as $\mathrm{Ge}$ and VS, respectively.

In Fig. 3, we report the PL spectra acquired at a constant pump power density of $4 \times 10^{5} \mathrm{~W} \mathrm{~cm}^{-2}$ and varying the lattice temperature from 80 to $330 \mathrm{~K}$ in $\sim 30-\mathrm{K}$ steps. The spectra acquired on Ge [Fig. 3(a)] show a broad peak at $\simeq 0.85 \mathrm{eV}$ at $80 \mathrm{~K}$, that redshifts to $\simeq 0.80 \mathrm{eV}$ at RT. We attribute this feature to the $\Gamma_{c}$-heavy-hole $\left(\Gamma_{c}-\mathrm{HH}\right)$ direct bandto-band recombination. PL experimental data associated with $\Gamma_{c}-\mathrm{HH}$ transitions have been fitted with the $T$ dependence of the direct gap, following the Varshni equation:

$$
E(T)=E(0)-\frac{\alpha T^{2}}{T+\beta}=0.868-\frac{5.82 \times 10^{-4} T^{2}}{T+296},
$$

where the parameters $\alpha$ and $\beta$ are those of bulk Ge [49] and $E(T)$ is in $\mathrm{eV}$. The behavior of the peaks as a function of temperature can be clearly observed in the contour plot of the PL spectra, reported in Fig. 3(b), where the fitting of the $\Gamma_{c}-\mathrm{HH}$ transitions is shown as a continuous white line. Increasing the temperature, the PL peak broadens and visually redshifts due to temperature-induced shrinking of the gap.

For comparison, we also report in Fig. 3(a) the spectral shape of the direct-gap recombination, obtained in the nondegenerate regime, following Ref. [48]:

$$
I(\hbar \omega)=\sqrt{\left(\hbar \omega-E_{g}\right)} \exp \left[-\left(\hbar \omega-E_{g}\right) / k_{B} T\right] .
$$

In Eq. (2), $E_{g}=0.769 \mathrm{eV}$ is the direct-gap energy, $k_{B}$ is the Boltzmann constant, and $T=450 \mathrm{~K}$ is the temperature of 


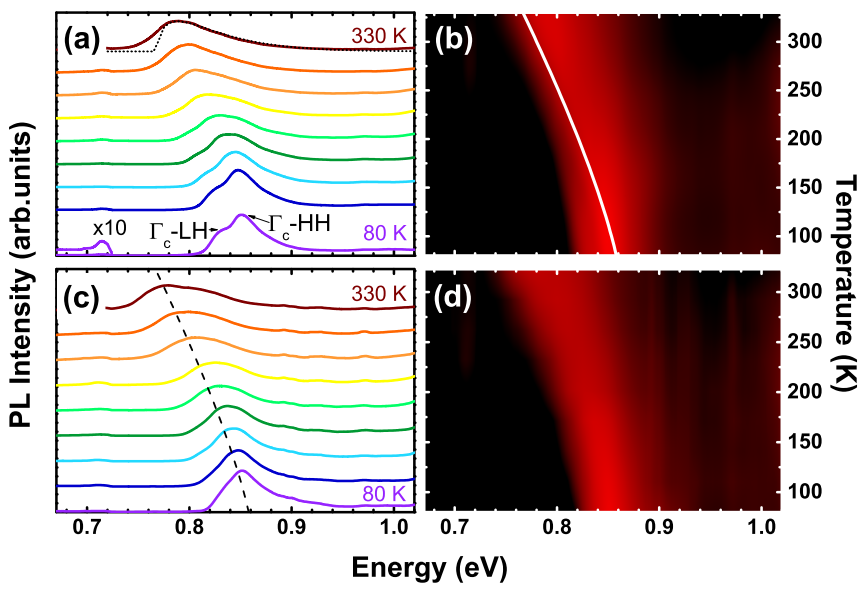

FIG. 3. Left: PL spectra acquired on (a) Ge and (c) VS. Lattice temperatures range from 80 to $330 \mathrm{~K}$ in $\sim 30-\mathrm{K}$ steps. The spectral shape of the direct transition at $330 \mathrm{~K}$ as given in Ref. [48] is also reported as dotted line in panel (a). The signal related to the indirect $L_{c}$-light-hole $\left(L_{c}-\mathrm{LH}\right)$ transition at $80 \mathrm{~K}$ in panel (a) has been enhanced by a factor of 10. Right: Contour plot of the PL spectra of (b) Ge and (d) VS as a function of $T$ with integrated intensity at each temperature normalized to unity. The results of the fitting of experimental data with Varshni equation are reported as continuous and dashed lines in panels (b) and (c), respectively.

the excited carriers, which is found to be higher than the lattice temperature of $T_{L}=330 \mathrm{~K}$ due to pump-induced electron heating effects.

On the low-energy side of the peaks acquired at low temperatures, we can see a shoulder related to the $\Gamma_{c}$ - $\mathrm{LH}$ direct recombination. As a matter of fact, for moderate inplane strain the LH-HH splitting $\delta$ is linearly dependent on the biaxial tensile strain $\varepsilon_{\|}$as $\delta=(6700 \pm 50) \mathrm{meV} \times$ $\varepsilon_{\|}[50,51]$. The in-plane strain arising from the differences in the thermal expansion coefficients $\alpha_{i}$ between $\mathrm{Ge}$ and $\mathrm{Si}$ during the cooling process from a high-temperature $T_{H}$ down to a lower-temperature $T_{L}$ is given by

$$
\varepsilon_{\|}\left(T_{L}, T_{H}\right) \simeq \int_{T_{L}}^{T_{H}}\left[\alpha_{\mathrm{Ge}}\left(T^{\prime}\right)-\alpha_{\mathrm{Si}}\left(T^{\prime}\right)\right] d T^{\prime} .
$$

From Eq. (3), it is clear that, decreasing the temperature $T_{L}$, the in-plane strain $\varepsilon_{\|}$increases, leading to a larger LH-HH splitting $\delta$. Assuming $T_{H} \simeq 875 \mathrm{~K} \mathrm{[47]} \mathrm{and} T_{L} \simeq 80 \mathrm{~K}, \varepsilon_{\|}$is estimated to be $\simeq 0.25 \%$ at $80 \mathrm{~K}$. The corresponding LH-HH splitting is $\simeq 17 \mathrm{meV}$, compatible with the peak separation in the PL spectra. At $300 \mathrm{~K}$, the biaxial tensile strain $\varepsilon_{\|}$calculated with Eq. (3) is reduced to $\varepsilon_{\|}=0.17$, matching the value obtained by XRD measurements. The corresponding LH-HH splitting $\delta$ is $\simeq 11 \mathrm{meV}$. Due to the reduced separation as well as the increased electron thermal energy, associated with a larger density of states for the $\mathrm{HH}$ band, the peak related to the direct $\Gamma_{c}$-LH cannot be clearly resolved. Indeed, the relative PL intensity of the two features in the temperature range investigated can be explained considering that, although the $\Gamma_{c}$-LH transition is energetically favored, the final density of states for the $\Gamma_{c}-\mathrm{HH}$ recombination and the associated dipole in the out-of-plane direction are larger than the corresponding quantities for the $\Gamma_{c}$-LH transition [41].
Finally, it is interesting to underline that the signal related to the indirect $L_{c}-\Gamma_{v}$ transition is much smaller at all the investigated temperatures [the weak signal at $\sim 0.71 \mathrm{eV}$ in Fig. 3(a), related to the $\Gamma_{c}-\mathrm{LH}$ transition, has been enhanced by a factor of 10], and the PL spectra are dominated by direct recombinations. The rationale is that, since we are dealing with epitaxial thin films, the optical path of the emitted light is small, and then the direct gap emission is not as much reabsorbed as in bulk Ge [52]. Moreover, in the whole investigated temperature range, excess electrons have sufficient thermal energy to populate the $\Gamma_{c}$ valley where the recombination rate is much higher [53].

The behavior of the PL spectra acquired on the VS sample [Fig. 3(c)] is similar to that observed in the Ge sample but, in this case, the high-energy side of the peaks is broader. Indeed, the fitting of the $\Gamma_{c}$-HH transitions in Ge with the Varshni equation (dashed line) reported in Fig. 3(c) clearly evidences the presence of a high-energy shoulder. Since the direct-gap energy of a $\mathrm{SiGe}$ alloy is an increasing function of its $\mathrm{Si}$ content, we can attribute this feature to the fact that we are also probing the direct recombination across the VS layers. In particular, our numerical results indicate that, at the pump energy used, the $95 \%$ step of the RG-VS also contributes to the PL signal, whereas the other GeSi layers, richer in $\mathrm{Si}$, remain almost transparent to the excitation (note that, as a consequence, reabsorption effects involving photons emitted from the inner $\mathrm{Ge}$ and $\mathrm{Ge}_{0.95} \mathrm{Si}_{0.05}$ layers can be also neglected in our samples).

Once that the origin of the peaks in the PL spectra has been established for the benchmark samples, temperaturedependent PL measurements have been carried out under the same conditions on sample S10-10. Since the photon energy is higher than the direct gap of Ge but smaller than the direct gap of the $\mathrm{Ge}_{0.85} \mathrm{Si}_{0.15}$ barriers, the quasiresonant excitation of carriers involves holes and electronic states confined in the Ge QWs.

PL spectra are shown in Fig. 4. At first glance, a clear difference between the sample S10-10 and the two benchmark samples is observed, consisting of the intense feature at high energy ranging from $\simeq 1 \mathrm{eV}$ at $80 \mathrm{~K}$ to $\simeq 0.9 \mathrm{eV}$ at $330 \mathrm{~K}$. Supported by our numerical model, we relate this feature to a direct transition inside the Ge well between the first confined states in the conduction and valence bands $\left(\Gamma_{0}-\mathrm{HH}_{0}\right)$. As for the direct recombination in $\mathrm{Ge}$, increasing the temperature, the $\Gamma_{0}-\mathrm{HH}_{0}$ transition is redshifted. At the same time, its intensity is quenched. On the low-energy side, a structure related to the indirect transition $L_{0}-\mathrm{HH}_{0}$ is also observed. Since this structure consists of two features separated by $\sim 56 \mathrm{meV}$ (arrows in Fig. 4), we attribute them to transitions accompanied either by emission or by absorption of a longitudinal acoustic phonon, being $\mathrm{E}_{L A}=28 \mathrm{meV}$ [52]. To support this attribution, we note that, increasing the temperature, the relative intensity of the peak related to phonon absorption is enhanced. The experimental and calculated energies of indirect and direct recombination energies are in excellent agreement as shown in Table II for selected temperatures.

In between the direct and the indirect transitions in the QWs, the direct transition $\left(\Gamma_{c}-\mathrm{HH}\right)$ due to the underlying VS is also observed. To better evidence the behavior, as a function of temperature, of the ratio between the intensity of 

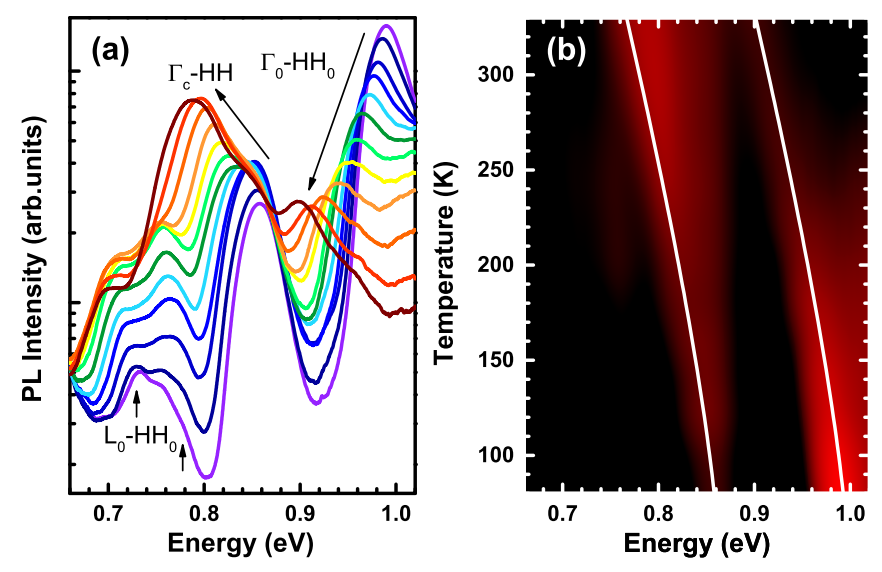

FIG. 4. (a) PL spectra acquired on sample S10-10. Lattice temperatures range from $80 \mathrm{~K}$ (violet) to $330 \mathrm{~K}$ (maroon) in $\sim 30-\mathrm{K}$ steps. (b) Contour plot of the PL spectra on sample S10-10 at different lattice temperatures. The integrated intensity at each temperature has been normalized to unity. The results of the fitting of experimental transitions in the Ge layer and the Ge QWs with the Varshni equation are also reported as continuous lines.

the PL feature related to the direct transition in the Ge well and the direct transition in the VS, we report in Fig. 4(b) a contour plot of the spectral intensity where the integrated intensity of each spectrum has been normalized to unity. From Fig. 4(b) it is clear that the intensity of the $\Gamma_{0}-\mathrm{HH}_{0}$ peak is quenched at increasing the temperature, whereas the intensity of the $\Gamma_{c}$-HH peak is boosted, the ratio between the two features going from $\simeq 4.39$ at $80 \mathrm{~K}$ to $\simeq 0.07$ at RT. Despite the $T$-dependent nonradiative recombination dynamics in the substrate and in the QW layers being largely undetermined, the observed behavior of the intensity ratio may suggest that the spatial distribution of the excess carrier density becomes more concentrated in the substrate region at increasing temperature.

To definitively confirm that the observed high-energy peak is related to the $\Gamma_{0}-\mathrm{HH}_{0}$ transition in the QW, we report in Fig. 5(b) the PL spectra acquired at $80 \mathrm{~K}$ on samples S10-2, S10-5, and S10-10 which have equal nominal thicknesses but different numbers of periods. Spectra have been normalized so that the intensity of the peak related to the direct transition in the VS is equal to unity. As for the XRD rocking curve, the position of the QW peak is the same for all the samples, indicating good repeatability and the absence of thickness fluctuations, whereas the intensity of the PL signal is approximately proportional to the number of QW periods as can be (a)

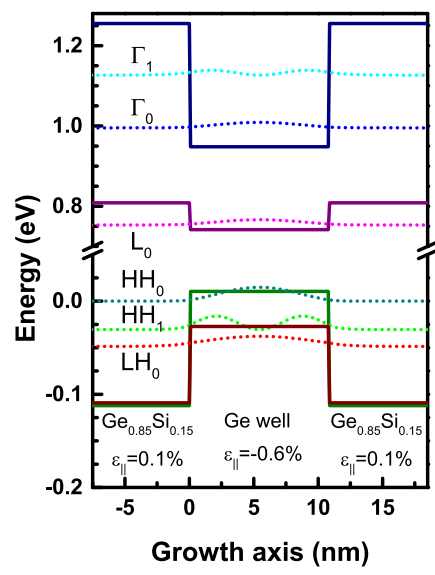

(b)

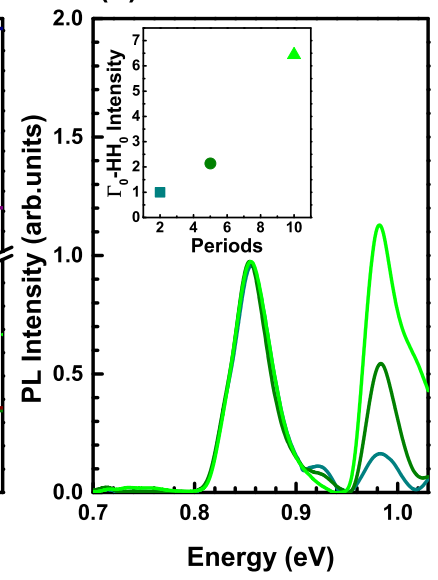

FIG. 5. (a) Conduction- and valence-band edge profiles (continuous lines) and square modulus of the wave functions (dotted lines) for the electron and hole confined states of sample S10-10 at $80 \mathrm{~K}$. (b) PL spectra acquired at $80 \mathrm{~K}$ on samples S10-2 (dark green), S10-5 (green), and S10-10 (light green). In the inset: Integrated PL intensity of the QWs peaks as a function of the number of periods.

seen in the inset of Fig. 5(b). This observation is compatible with a scenario where the MQWs are uniformly excited and the ratio between the excess carrier density in a single QW and in the substrate does not vary significantly with the number of periods.

To quantitatively characterize the direct band transition in the Ge wells of sample S10-10, we have calculated the corresponding electronic states and band structure [see Fig. 5(a)]. The experimental and theoretical energies for $\Gamma_{0}-\mathrm{HH}_{0}$ as a function of the temperature are reported in Fig. 6(a) as filled and empty circles, respectively. Experimental data have been fitted following the Varshni equation with the same values for $\alpha$ and $\beta$ used in Eq. (1) but setting a larger $E(0)$ to account for the confinement energy. The result of this fitting procedure is reported in Fig. 6 as a continuous line. The experimental, calculated, and fitted energies are in good agreement, confirming that this PL feature originates from direct transitions in the $\mathrm{Ge}$ QWs involving the fundamental $\mathrm{HH}_{0}$ and $\Gamma_{0}$ confined states.

In the right panel of Fig. 6 we show the integrated intensity of the PL spectra as a function of $\beta=1 / k_{B} T$. The integrated intensity, collected from the Ge sample, is displayed as squares, whereas circles represent the intensity of the QWs feature in sample S10-10. For both samples, data in Fig. 6 have been normalized to unity at $80 \mathrm{~K}$. The two curves show a nonmonotonic trend with a single minimum at $\beta$ equal to

TABLE II. Experimental and calculated $\Gamma_{0}-\mathrm{HH}_{0}$ and $L_{0}-\mathrm{HH}_{0}$ transition energies at selected temperatures.

\begin{tabular}{|c|c|c|c|c|c|}
\hline \multirow[b]{2}{*}{ Temperature $(\mathrm{K})$} & \multicolumn{2}{|c|}{$\Gamma_{0}-\mathrm{HH}_{0}(\mathrm{eV})$} & \multicolumn{3}{|c|}{$L_{0}-\mathrm{HH}_{0}(\mathrm{eV})$} \\
\hline & Experimental & Theory & Experimental (phonon emission) & Experimental (phonon absorption) & Theory \\
\hline 80 & $0.990 \pm 0.002$ & 0.995 & $0.733 \pm 0.002$ & $0.779 \pm 0.002$ & 0.753 \\
\hline 140 & $0.977 \pm 0.002$ & 0.980 & $0.716 \pm 0.002$ & $0.765 \pm 0.002$ & 0.738 \\
\hline 200 & $0.958 \pm 0.002$ & 0.959 & $0.708 \pm 0.002$ & $0.758 \pm 0.002$ & 0.719 \\
\hline 300 & $0.913 \pm 0.002$ & 0.916 & & $0.706 \pm 0.002$ & 0.682 \\
\hline
\end{tabular}



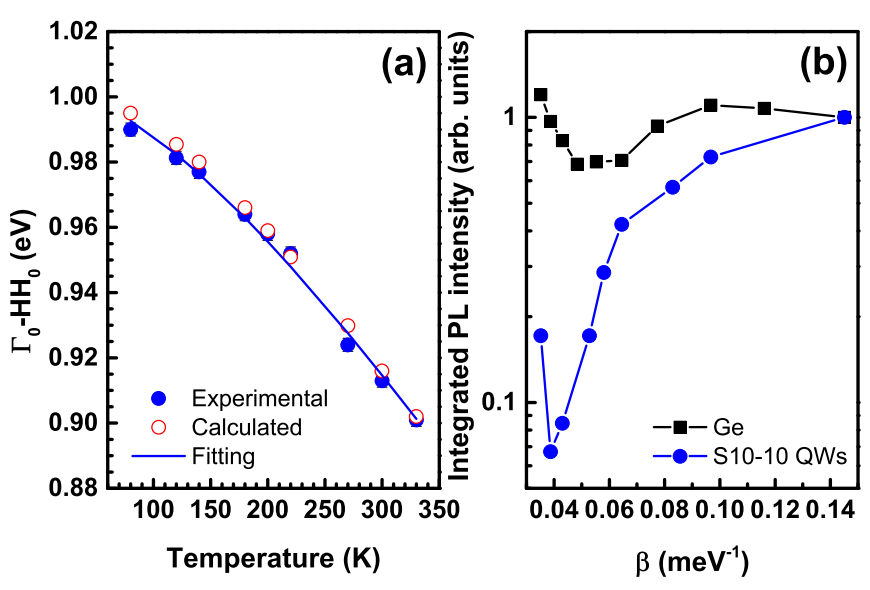

FIG. 6. (a) Experimental (filled circles) and calculated (empty circles) energy of the $\Gamma_{0}-\mathrm{HH}_{0}$ transition in sample $\mathrm{S} 10-10$ as a function of the lattice temperature. The fitting of the experimental data with the Varshni equation is reported with a continuous line. (b) Integrated PL intensity as a function of $\beta=1 / k_{B} T$. Squares and circles represent $\mathrm{Ge}$ and sample $\mathrm{S} 10-10$, respectively.

0.048 and 0.039 for the Ge and QW samples, respectively. This behavior can be attributed to the interplay between two different regimes. In the high-temperature regime, the negative slope is determined by the thermal boost of the PL intensity induced by the increase in the electron population in the $\Gamma$ valley $[15,53]$ and by the thermal emission of carriers from dislocations [54]. The quenching of the PL dominating in the low- $T$ regime is instead caused by nonradiative processes whose rates increase with $T$ [55-57]. In the case of the QW sample, the thermal promotion of electrons from $L_{0}$ to $\Gamma_{0}$ is hindered by the larger energy difference occurring between the direct and the indirect gaps, which in the QW system is associated with the lighter confinement mass of $\Gamma$ electrons with respect to the $L$ ones. Therefore in the high- $T$ regime, the increase with $T$ of the nonradiative recombination rate plays in this case a major role in suppressing the PL signal. Moreover, also the $T$-driven migration of excess carriers toward the substrate region observed increasing $T$ [see Fig. 4(b)] contribute to the quenching of the $\Gamma_{0}-\mathrm{HH}_{0}$ signal. As a result, the integrated PL signal for the QW sample shown in Fig. 6 as a function of $\beta$ spans a broader range, and the minimum is shifted to a lower value with respect to the $\mathrm{Ge}$ case.

To clarify the effect of quantum confinement, we also performed temperature-dependent PL measurements on QWs with different thicknesses, whose values have been measured by XRD and TEM (see Table I). The experimental and calculated $\Gamma_{0}-\mathrm{HH}_{0}$ transition energies at $80 \mathrm{~K}$ and $\mathrm{RT}$ are reported as a function of the QW thickness in Fig. 7 as filled and empty symbols, respectively. Their values are larger than the one associated with the direct recombination in the $\mathrm{Ge}$ sample ( $\simeq 0.85 \mathrm{eV}$ at $80 \mathrm{~K}$ and $\simeq 0.80 \mathrm{eV}$ at $\mathrm{RT})$ due to the concomitant effect of quantum confinement and compressive strain. Moreover, as expected, a redshift of the PL peak with the increase in the well thickness is clearly observed.

Finally, we conclude discussing PL data collected at different pump-power densities. In Fig. 8(a), we show PL spectra

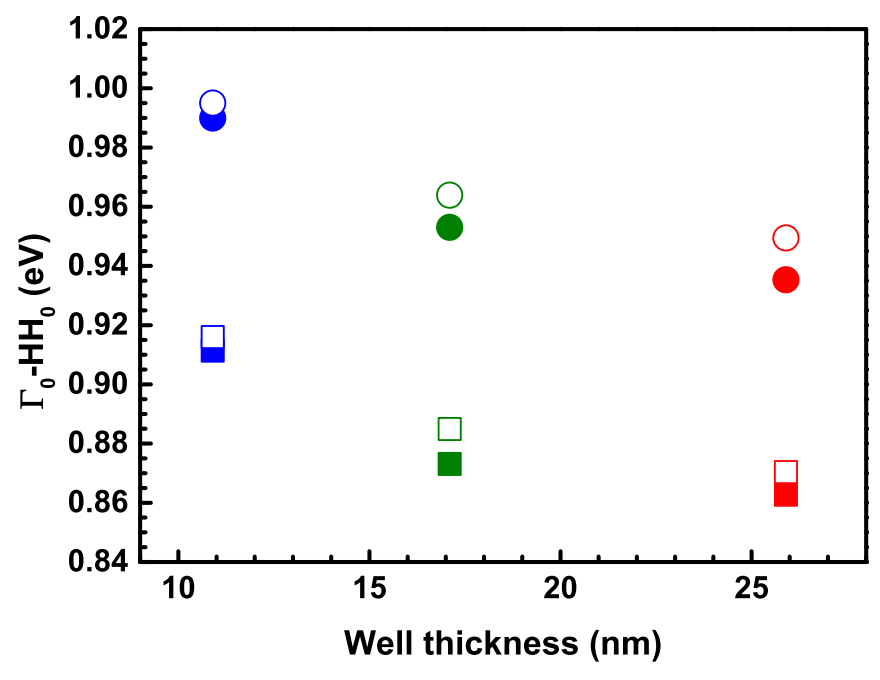

FIG. 7. Experimental (filled symbols) and calculated (empty symbols) energy of the $\Gamma_{0}-\mathrm{HH}_{0}$ transition as a function of the QWs thickness at $80 \mathrm{~K}$ (circles) and $300 \mathrm{~K}$ (squares).

measured from the $\mathrm{Ge}$ sample at $80 \mathrm{~K}$ in the $5.6 \times 10^{4}$ and $5.6 \times 10^{5}-\mathrm{W} \mathrm{cm}^{-2}$ ranges. Note that the peak position is not redshifted at high-power density, pointing to the absence of significative pump-induced lattice heating. The LH-HH splitting is clearly observed in each curve and, increasing the pump power density, the relative intensity of the $\Gamma_{c}-\mathrm{HH}$ recombination increases with respect to the $\Gamma_{c}$ - $\mathrm{LH}$ one due to the larger density of hole states.

PL spectra, as a function of the laser pump power density, measured on sample S25-5, with $t_{w}=25.9 \mathrm{~nm}$, are reported in Fig. 8(b). Again, increasing the power density, the energies of the $\Gamma_{c}-\mathrm{HH}$ and $\Gamma_{c}-\mathrm{LH}$ recombinations in the Ge layer are not affected, whereas the peak related to the $\Gamma_{0}-\mathrm{HH}_{0}$ transition slightly redshifts at excitation densities
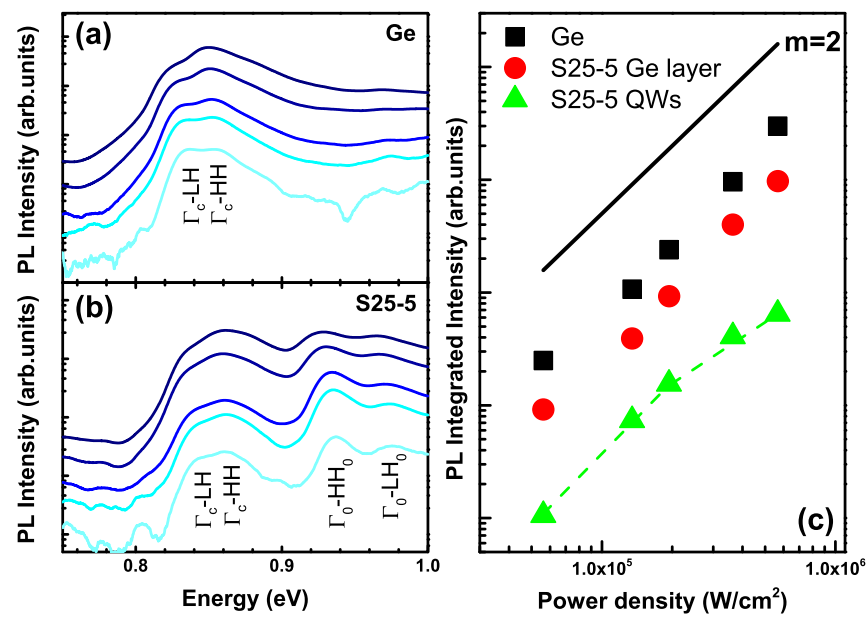

FIG. 8. Left: PL spectra measured from the (a) Ge and (b) S25-5 samples at $80 \mathrm{~K}$ with different pump power densities, ranging in the $5.6 \times 10^{4}-5.6 \times 10^{5}-\mathrm{W} \mathrm{cm}^{-2}$ interval. Right: Integrated PL intensity for the Ge sample (square) and for the Ge (circle) and QW (triangle) features of the S25-5 sample as a function of the pump-power density. 
of $>1.9 \times 10^{5} \mathrm{~W} \mathrm{~cm}^{-2}$. Interestingly, in this larger-well sample, a spectral feature at $\sim 39 \mathrm{meV}$ above the $\Gamma_{0}-\mathrm{HH}_{0}$ one is also distinguishable. Since our model predicts an excess energy of $40 \mathrm{meV}$ for the $\Gamma_{0}-\mathrm{LH}_{0}$ recombination, we can safely attribute this additional peak to radiative recombinations across the direct gap, involving the light-hole fundamental state.

Figure 8(c) shows the integrated PL intensity at $80 \mathrm{~K}$ as a function of the excitation power density. Data of the Ge sample and the component related to the Ge layer in S25-5 follow a power-law dependence $I \propto W^{m}$. The fit-power exponents $m$ found are close to the theoretical value of $m=2$ (black line), expected when the dominant nonradiative mechanism is related to the Shockley-Read-Hall recombination [53]. On the other hand, the integrated intensity of the QWs' feature shows a scaling exponent $m=2$ for excitation densities up to $1.9 \times 10^{5} \mathrm{~W} \mathrm{~cm}^{-2}$ but, increasing further the excitation, the intensity tends to level off to a value of $m \simeq 1.3$ indicating the contribution of Auger recombination mechanism.

\section{CONCLUSIONS}

To summarize, we have analyzed, through microphotoluminescence measurements and theoretical calculations, the optical properties of undoped strain-unbalanced Ge MQWs surrounded by Ge-rich GeSi barriers, grown on reversegraded GeSi virtual substrates by means of ultrahigh-vacuum chemical vapor deposition. In view of the exploitation of $\mathrm{Ge} / \mathrm{GeSi} \mathrm{MQWs}$ as optical emitters, these results are crucial to unambiguously understand the photoluminescence spectra of samples with few periods of QWs, grown on reversegraded virtual substrates and featuring an external tensile stressor layer. The high quality of the samples has been confirmed by high-resolution transmission electron microscopy, $\mathrm{x}$-ray diffraction, and atomic force microscopy. The structural analysis demonstrates that strain symmetrization is not a mandatory requirement for few multilayer repetitions. The good agreement between experimental data and theoretically predicted transition energies validates the proposed modeling and allows us to distinguish the spectral features originating in the excited portion of the substrate from those associated to the QWs.

\section{ACKNOWLEDGMENTS}

Part of this work was supported by the European Union Research and Innovation programme Horizon 2020 under Grant No. 766719—FLASH Project.
[1] Y. H. Kuo, Y. K. Lee, Y. Ge, S. Ren, J. E. Roth, T. I. Kamins, D. A. B. Miller, and J. S. Harris, Nature (London) 437, 1334 (2005).

[2] D. J. Paul, Phys. Rev. B 77, 155323 (2008).

[3] R. K. Schaevitz, J. E. Roth, S. Ren, O. Fidaner, and D. A. B. Miller, IEEE J. Sel. Top. Quantum Electron. 14, 1082 (2008).

[4] D. Marris-Morini, P. Chaisakul, M.-S. Rouifed, J. Frigerio, D. Chrastina, G. Isella, S. Edmond, X. Le Roux, J.-R. Coudevylle, and L. Vivien, Nanophotonics 2, 279 (2013).

[5] P. Chaisakul, D. Marris-Morini, G. Isella, D. Chrastina, M. Rouifed, J. Frigerio, and L. Vivien, Solid-State Electron. 83, 92 (2013).

[6] L. Lever, Y. Hu, M. Myronov, X. Liu, N. Owens, F. Y. Gardes, I. P. Marko, S. J. Sweeney, Z. Ikonić, D. R. Leadley, G. T. Reed, and R. W. Kelsall, Opt. Lett. 36, 4158 (2011).

[7] O. Fidaner, A. K. Okyay, J. E. Roth, R. K. Schaevitz, Y. H. Kuo, K. C. Saraswat, J. S. Harris, and D. A. B. Miller, IEEE Photonics Technol. Lett. 19, 1631 (2007).

[8] E. Onaran, M. C. Onbasli, A. Yesilyurt, H. Y. Yu, A. M. Nayfeh, and A. K. Okyay, Opt. Express 20, 7608 (2012).

[9] G.-E. Chang, S.-W. Chen, and H. H. Cheng, Opt. Express 24, 17562 (2016).

[10] C. Lange, G. Isella, D. Chrastina, F. Pezzoli, N. S. Köster, R. Woscholski, and S. Chatterjee, Phys. Rev. B 85, 241303 (2012).

[11] A. Giorgioni, F. Pezzoli, E. Gatti, S. Cecchi, C. Kazuo Inoki, C. Deneke, E. Grilli, G. Isella, and M. Guzzi, Appl. Phys. Lett. 102, 012408 (2013).

[12] A. Giorgioni, S. Paleari, S. Cecchi, E. Vitiello, E. Grilli, G. Isella, W. Jantsch, M. Fanciulli, and F. Pezzoli, Nat. Commun. 7, 13886 (2016).

[13] S. De Cesari, E. Vitiello, A. Giorgioni, and F. Pezzoli, Electronics 6, 19 (2017).
[14] P. Chaisakul, D. Marris-Morini, G. Isella, D. Chrastina, N. Izard, X. Le Roux, S. Edmond, J.-R. Coudevylle, and L. Vivien, Appl. Phys. Lett. 99, 141106 (2011).

[15] E. Gatti, E. Grilli, M. Guzzi, D. Chrastina, G. Isella, and H. von Känel, Appl. Phys. Lett. 98, 031106 (2011).

[16] J. Jiang and J. Sun, Opt. Express 24, 14525 (2016).

[17] J. Liu, X. Sun, R. Camacho-Aguilera, L. C. Kimerling, and J. Michel, Opt. Lett. 35, 679 (2010).

[18] M. J. Süess, R. Geiger, R. A. Minamisawa, G. Schiefler, J. Frigerio, D. Chrastina, G. Isella, R. Spolenak, J. Faist, and H. Sigg, Nat. Photonics 7, 466 (2013).

[19] S. Bao, D. Kim, C. Onwukaeme, S. Gupta, K. Saraswat, K. H. Lee, Y. Kim, D. Min, Y. Jung, H. Qiu, H. Wang, E. A. Fitzgerald, C. S. Tan, and D. Nam, Nat. Commun. 8, 1845 (2017).

[20] D. S. Sukhdeo, D. Nam, J.-H. Kang, M. L. Brongersma, and K. C. Saraswat, Photonics Res. 2, A8 (2014).

[21] J. R. Jain, A. Hryciw, T. M. Baer, D. A. B. Miller, M. L. Brongersma, and R. T. Howe, Nat. Photonics 6, 398 (2012).

[22] M. Bollani, D. Chrastina, L. Gagliano, L. Rossetto, D. Scopece, M. Barget, V. Mondiali, J. Frigerio, M. Lodari, F. Pezzoli, F. Montalenti, and E. Bonera, Appl. Phys. Lett. 107, 083101 (2015).

[23] A. Ghrib, M. El Kurdi, M. Prost, S. Sauvage, X. Checoury, G. Beaudoin, M. Chaigneau, R. Ossikovski, I. Sagnes, and P. Boucaud, Adv. Opt. Mater. 3, 353 (2015).

[24] G. Capellini, G. Kozlowski, Y. Yamamoto, M. Lisker, C. Wenger, G. Niu, P. Zaumseil, B. Tillack, A. Ghrib, M. de Kersauson, M. El Kurdi, P. Boucaud, and T. Schroeder, J. Appl. Phys. 113, 013513 (2013).

[25] A. Ghrib, M. de Kersauson, M. El Kurdi, R. Jakomin, G. Beaudoin, S. Sauvage, G. Fishman, G. Ndong, M. Chaigneau, 
R. Ossikovski, I. Sagnes, and P. Boucaud, Appl. Phys. Lett. 100, 201104 (2012).

[26] D. S. Sukhdeo, J. Petykiewicz, S. Gupta, D. Kim, S. Woo, Y. Kim, J. Vučković, K. C. Saraswat, and D. Nam, Opt. Express 23, 33249 (2015).

[27] G. Capellini, C. Reich, S. Guha, Y. Yamamoto, M. Lisker, M. Virgilio, A. Ghrib, M. E. Kurdi, P. Boucaud, B. Tillack, and T. Schroeder, Opt. Express 22, 399 (2014).

[28] G. A. Chahine, M. H. Zoellner, M.-I. Richard, S. Guha, C. Reich, P. Zaumseil, G. Capellini, T. Schroeder, and T. U. Schülli, Appl. Phys. Lett. 106, 071902 (2015).

[29] Y. Chen, C. Li, Z. Zhou, H. Lai, S. Chen, W. Ding, B. Cheng, and Y. Yu, Appl. Phys. Lett. 94, 141902 (2009).

[30] Y. Chen, C. Li, H. Lai, and S. Chen, Nanotechnology 21, 115207 (2010).

[31] L. M. Giovane, H.-C. Luan, A. M. Agarwal, and L. C. Kimerling, Appl. Phys. Lett. 78, 541 (2001).

[32] J. G. Fiorenza, G. Braithwaite, C. W. Leitz, M. T. Currie, J. Yap, F. Singaporewala, V. K. Yang, T. A. Langdo, J. Carlin, M. Somerville, A. Lochtefeld, H. Badawi, and M. T. Bulsara, Semicond. Sci. Technol. 19, L4 (2004).

[33] A. D. Capewell, T. J. Grasby, T. E. Whall, and E. H. C. Parker, Appl. Phys. Lett. 81, 4775 (2002).

[34] V. Shah, A. Dobbie, M. Myronov, and D. Leadley, Thin Solid Films 520, 3227 (2012).

[35] G. Capellini, M. De Seta, Y. Busby, M. Pea, F. Evangelisti, G. Nicotra, C. Spinella, M. Nardone, and C. Ferrari, J. Appl. Phys. 107, 063504 (2010).

[36] E. A. Fitzgerald, Y.-H. Xie, M. L. Green, D. Brasen, A. R. Kortan, J. Michel, Y.-J. Mii, and B. E. Weir, Appl. Phys. Lett. 59, 811 (1991).

[37] M. Virgilio and G. Grosso, Phys. Rev. B 77, 165315 (2008).

[38] G. Pizzi, M. Virgilio, and G. Grosso, Nanotechnology 21, 055202 (2010).

[39] T. Wendav, I. A. Fischer, M. Virgilio, G. Capellini, F. Oliveira, M. F. Cerqueira, A. Benedetti, S. Chiussi, P. Zaumseil, B. Schwartz, K. Busch, and J. Schulze, Phys. Rev. B 94, 245304 (2016).

[40] D. Sabbagh, J. Schmidt, S. Winnerl, M. Helm, L. Di Gaspare, M. De Seta, M. Virgilio, and M. Ortolani, ACS Photonics 3, 403 (2016).

[41] M. Virgilio, M. Bonfanti, D. Chrastina, A. Neels, G. Isella, E. Grilli, M. Guzzi, G. Grosso, H. Sigg, and H. von Känel, Phys. Rev. B 79, 075323 (2009).
[42] Y. Busby, M. De Seta, G. Capellini, F. Evangelisti, M. Ortolani, M. Virgilio, G. Grosso, G. Pizzi, P. Calvani, S. Lupi, M. Nardone, G. Nicotra, and C. Spinella, Phys. Rev. B 82, 205317 (2010).

[43] M. R. Barget, M. Virgilio, G. Capellini, Y. Yamamoto, and T. Schroeder, J. Appl. Phys. 121, 245701 (2017).

[44] E. Fitzgerald and S. Samavedam, Thin Solid Films 294, 3 (1997).

[45] M. H. Zoellner, M.-I. Richard, G. A. Chahine, P. Zaumseil, C. Reich, G. Capellini, F. Montalenti, A. Marzegalli, Y.-H. Xie, T. U. Schülli, M. Häberlen, P. Storck, and T. Schroeder, ACS Appl. Mater. Interfaces 7, 9031 (2015).

[46] M.-I. Richard, M. H. Zoellner, G. A. Chahine, P. Zaumseil, G. Capellini, M. Häberlen, P. Storck, T. U. Schülli, and T. Schroeder, ACS Appl. Mater. Interfaces 7, 26696 (2015).

[47] G. Capellini, M. De Seta, P. Zaumseil, G. Kozlowski, and T. Schroeder, J. Appl. Phys. 111, 073518 (2012).

[48] H. B. Bebb and E. W. Williams, Semicond. Semimetals 8, 181 (1972).

[49] L. E. Vorobyev, in Handbook Series on Semiconductor Parameters, edited by M. E. Levinshtein, S. L. Rumyantsev, and M. Shur (World Scientific, London, 1996), Vol. 1, Chap. 2, pp. 33-57.

[50] L. Carroll, F. Imbert, H. Sigg, M. Süess, E. Müller, M. Virgilio, G. Pizzi, P. Rossbach, D. Chrastina, and G. Isella, Appl. Phys. Lett. 99, 031907 (2011).

[51] E. Vitiello, M. Virgilio, A. Giorgioni, J. Frigerio, E. Gatti, S. De Cesari, E. Bonera, E. Grilli, G. Isella, and F. Pezzoli, Phys. Rev. B 92, 201203 (2015).

[52] T. Arguirov, M. Kittler, and N. V. Abrosimov, J. Phys.: Conf. Ser. 281, 012021 (2011).

[53] M. Virgilio, T. Schroeder, Y. Yamamoto, and G. Capellini, J. Appl. Phys. 118, 233110 (2015).

[54] F. Pezzoli, A. Giorgioni, K. Gallacher, F. Isa, P. Biagioni, R. W. Millar, E. Gatti, E. Grilli, E. Bonera, G. Isella, D. J. Paul, and L. Miglio, Appl. Phys. Lett. 108, 262103 (2016).

[55] M. Virgilio, C. L. Manganelli, G. Grosso, T. Schroeder, and G. Capellini, J. Appl. Phys. 114, 243102 (2013).

[56] F. Pezzoli, F. Isa, G. Isella, C. V. Falub, T. Kreiliger, M. Salvalaglio, R. Bergamaschini, E. Grilli, M. Guzzi, H. von Känel, and L. Miglio, Phys. Rev. Appl. 1, 044005 (2014).

[57] F. Pezzoli, A. Giorgioni, D. Patchett, and M. Myronov, ACS Photonics 3, 2004 (2016). 